\title{
Design Improvements of a Silicon Photonic ROADM (Project Report CIAN2-1-Y4)
}

\section{SHAYAN MOOKHERJEA}

Department of Electrical and Computer Engineering, University of California, San Diego, MC 0407 La Jolla CA 92093-0407 USA

Email:smookher@ucsd.edu

\section{Introduction and Goals}

A partnership between UC San Diego, Columbia University, UC Berkeley, Caltech and the University of Arizona collaborated with Sandia National Labs in a second NSF-funded silicon photonics multi-project wafer (MPW) project in 2016.

This was a project "Silicon Photonics Device Manufacturing and Test" under the re-organized Thrust 2 "Subsystem Integration and Silicon Nanophotonics" of the NSF-funded Center for Integrated Access Networks. The goal of this research was to design, fabricate and test chip-scale opto-electronic devices that can be useful in access and data networks. Designs were submitted for manufacturing in 2015 and diced microchips were returned to the designers for testing in 2016 - 2017. This is a report of the $2^{\text {nd }}$ generation ROADM (reconfigurable optical add drop multiplexer) device made using silicon photonics, including passive and doped silicon waveguides and metalization.

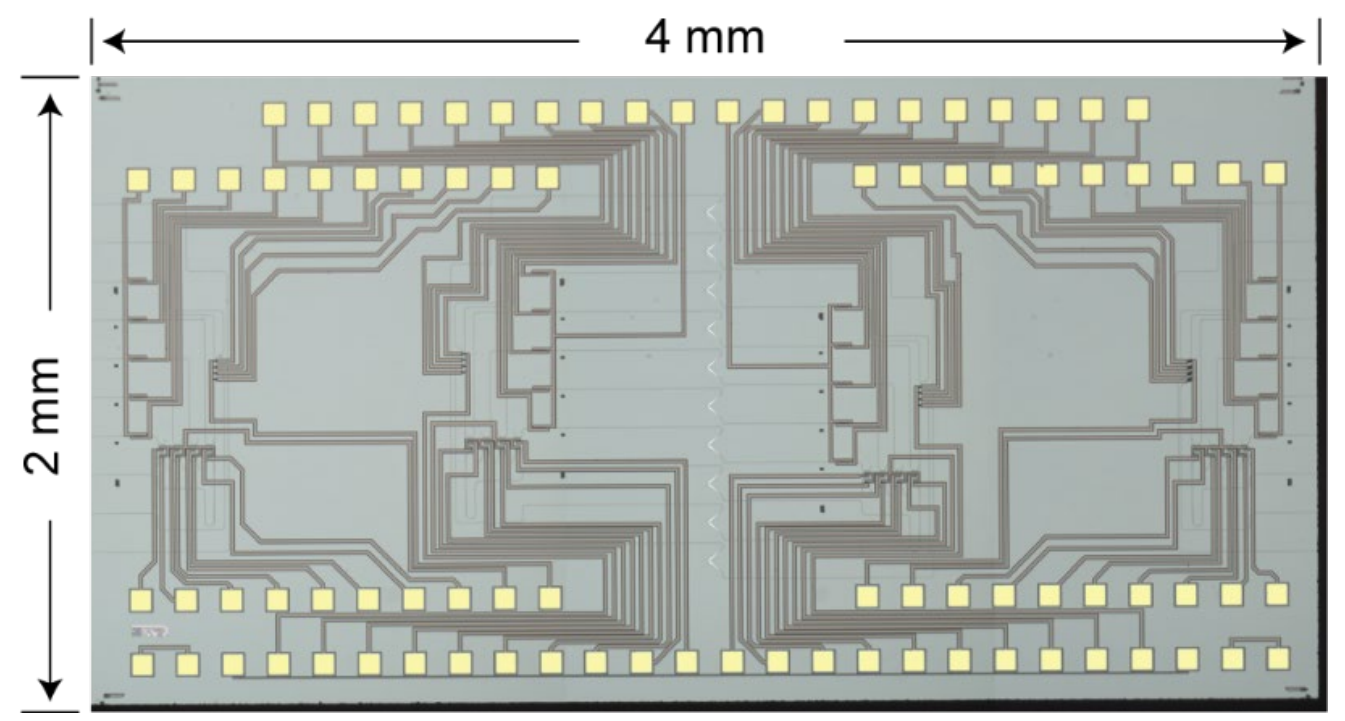

Figure 1 Image of a second-generation silicon photonic add-drop multiplexer chip which addresses four WDM channels. A group of four WDM channels (100 GHz spacing) can be added or dropped from the bus waveguide. Variable optical attenuators (VOAs) are also included for each of the four channels as well as the bus input. Edge couplers are located at the east and west edges of the chip. Grating couplers located near the middle of the chip were used for the input and output channels. Four independent ROADM test designs are located on this die. 


\section{Activities}

Figure 1 shows an optical microscope image of the fabricated test microchip [1]. Electrical contact pads are situated along the perimeter of the chip and the optical circuitry is in the middle. Some chips were die-attached to carriers and wire-bonded for ease of measurement. Edge couplers to fiber are located along the east and west edges of the chip. Fiber-attach was not performed in this project. However, we used an array of high numerical aperture fibers to perform some of the measurements. A set of grating couplers is located near the middle of the chip for diagnostic measurements.

The chip serves the same purpose as the first-generation design: a group of four C-band WDM channels (externally modulated with $10 \mathrm{Gbit} / \mathrm{s}$ NRZ data from commercial transceivers at fixed wavelengths) could be added or dropped from a bus waveguide (also called "express channel") which contains up to 24 WDM channels. This chip was intended for use in a campus ring network (UCSD "MORDIA") using for networking research at UC San Diego [2]. The add-drop functionality was provided using coupled-microring filters. The channels were tunable using thermo-optic tuning of the microrings. In addition, the power level of each input could be electronically controlled on sub-microsecond speeds using a variable optical attenuator (VOA). Off-chip lasers were used for testing. No packaging or driver integration was performed.

The basic principles of the design are the same as the first-generation design. The optical filtering structures were implemented using single or coupled silicon microrings. Previously, we had experimentally studied the dispersion of directional couplers using a microring coupled to a waveguide [3]. An accurate knowledge of dispersion is also necessary for designing coupled-microring filters that are part of more complex designs, such as the combination of wavelength conversion and optical filtering [4].

\section{Progress and Impact}

Figure 2 shows measurements of the four-channel drop functionality of the $2^{\text {nd }}$ generation microchip compared to the earlier version. The drop port was routed to a defined output port so that the transmission spectra could be measured. As before, four microring resonators were used to achieve the drop functionality. The microrings have a small radius, so that the free spectral range (FSR) is greater than $35 \mathrm{~nm}$ and only one resonance appears in the C-band. The filter response was not designed to achieve the perfect brick-wall shape but was improved from the $1^{\text {st }}$ generation design. The transmission contrast was greater than $15 \mathrm{~dB}$. Each of the four channels was filtered by a thermo-optically tuned coupled-microring filter bank. The heaters were redesigned to be more robust and withstood the higher voltages needed to tune over a wider range of 
wavelengths. About $30 \mathrm{~nm}$ tuning range could be covered before the heaters were damaged from excessive power dissipation. This design is sensitive to the exact gap between the ring and the waveguide, and therefore benefits from iterative fabrication attempts. However, several aspects of the fabrication process changed between 2014 and 2016, and therefore, the fabricated device was not exactly as intended. Nevertheless, the performance was a substantial improvement over the first-generation device.
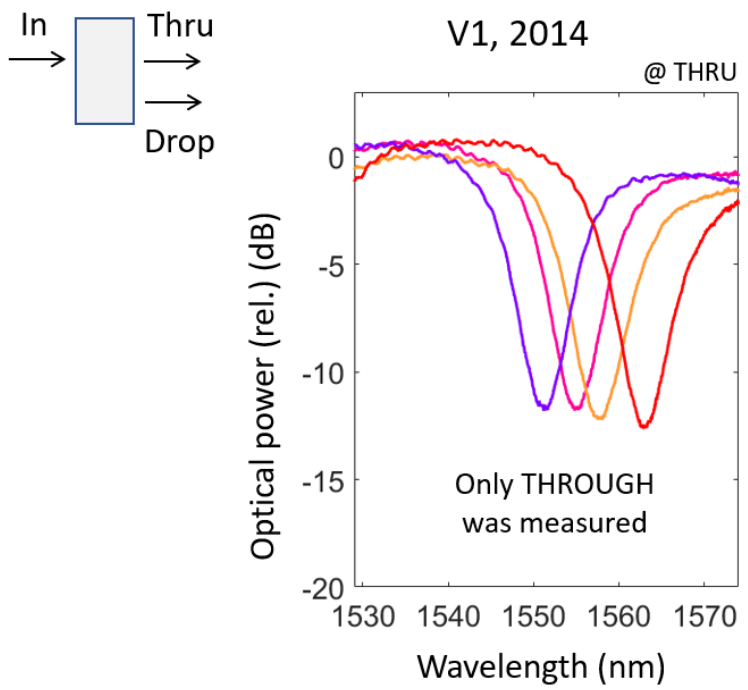

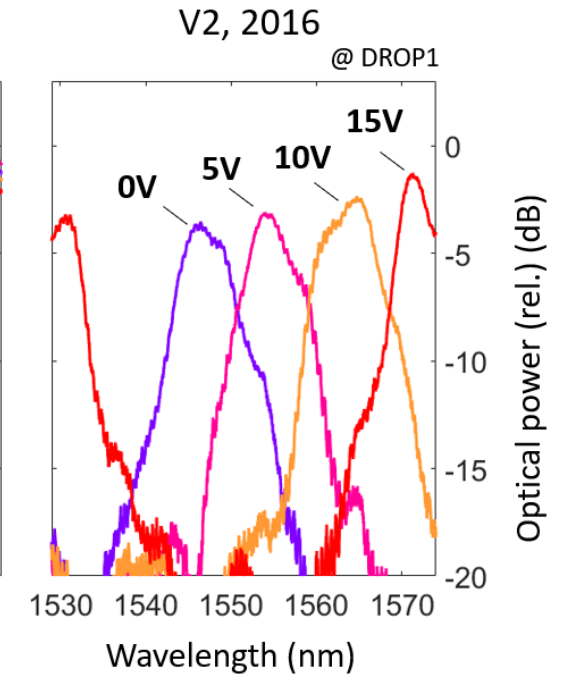

V2, 2016

Figure 2 Improved drop response in the second-generation design, including the ability to tune over a wider range of wavelengths. In this design, the drop ports were more easily measurable than in the first-generation design.

Figure 3 shows measurements of the four-channel add functionality. The target FSR was $800 \mathrm{GHz}$, and the experimentally achieved FSR was $850 \mathrm{GHz}$. This is implemented after the drop section, and therefore, larger silicon microrings with a lower FSR can be used. Each channel is added using a coupledmicroring add-drop filter using silicon microring resonators. A substantially improved channel-to-channel separation was measured. As before, heaters were incorporated into the microrings, by doping the inner radius of the waveguide bends. In the revised designs, a more careful design and simulation study was undertaken, including an estimation of the diffusion of dopants through the anneal process. Dopants placed too close to the optical mode will result a high optical loss and poor filter function. We suspect that the first-generation devices had a much higher optical loss than desirable.

As an alternative, metal heaters can be used which are formed using serpentine bends of metal traces above or near the silicon microresonator, but not as part of the same structure. These structures, which we studied in other projects, are more robust and also result in lower optical loss. However, the filters cannot be tuned as rapidly, which may be a concern in some applications. 


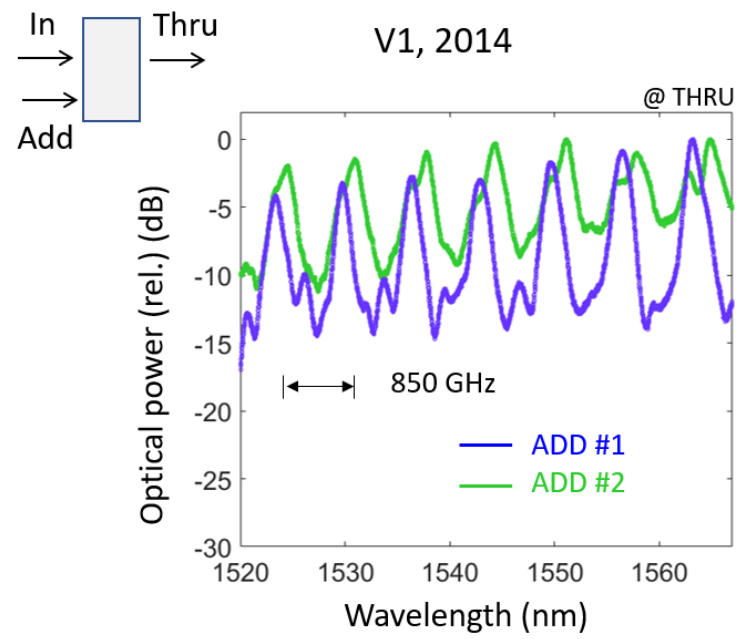

V2, 2016

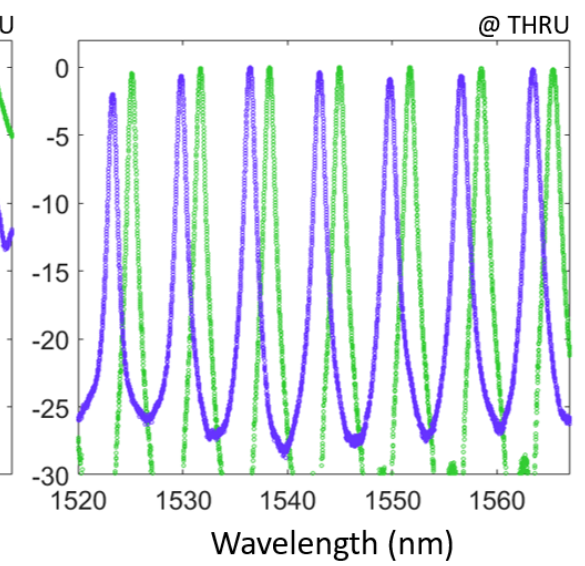

Figure 3 Improved add response (shown here for 2 channels) in the second-generation design. Double ring resonators were used for each add channel. An improved channel-to-channel separation and cleaner filter response were measured.

These results clearly show that a substantial improvement was achieved in the optical device design for the drop and add filter sections. We were able to perform detailed measurements on a first batch of deliverables, and use those results in making the next generation of the design. Each iteration took about 2 years from design to measurement, and cost of fabrication is also high for each run. Thus, only the larger NSF-funded projects can support the expenses and extended timeline of multiple fabrication runs.

Coupled microrings were used to achieve an improved filtering response compared to single microring resonators when integrated as part of a more diverse photonic circuit. At around the same time as this project, we fabricated fifth-order coupled-resonator filters in another project with even better performance [5]. This shows a roadmap towards even better performance in the future, with a more flat top response, steeper sidewalls, and better tolerance to disorder. Previously, we have developed the theory and design principles of coupled microring resonators, also known as Coupled Resonator Optical Waveguides (CROWs). A tight-binding model of band-center propagation was formulated [6], and theoretical studies of nonlinear propagation were developed on this basis [7], [8]. Studies were performed to examine the regions of high dispersion which may be relevant to signal distortion when filtering wideband signals [9] or all-optically tunable dispersion compensation devices [10]. To help in designing structures in silicon photonics, we have developed an extension of coupled-mode theory which is applicable to directional couplers in high index contrast materials, in coupled-waveguide structures [11] and coupled-resonator structures [12]. 
An NSF-supported collaboration with IBM's silicon photonics group (Dr. W. M. J. Green) has provided insights into silicon photonics fabrication and also supported technical collaboration on fabricating structures at wafer-scale using silicon photonics processing [13], [14]. Dr. Ivan B. Divliansky (CREOL, Florida) collaborated with us for the electron-beam lithography of long waveguide structures [15]. CROWs of up to 235 coupled resonators were successfully fabricated and measured, and a good agreement was achieved between experiments and theory [14], [16], [17].

Impact on Human Resources: Two graduate students in the PI's group worked on this topic as part of their education and training. Other students contributed to the microchip measurements and preparing papers for publication. The Center provided valuable opportunities to interact with researchers and students from other groups and universities as well as with researchers from Sandia and from industry.

\section{Subsequent Extensions}

CROW structures can have other applications in photonics, such as the generation of entangled photon pairs [18], and developing novel amplifiers and lasers [19]. For example, the resonator-enhanced nonlinearity benefits the generation of entangled photon-pair and heralded single-photon generation using silicon photonics [20]. Our initial measurements of spontaneous fourwave mixing (SFWM) were on correlated photon-pair generation and heralded single-photon generation. An experimental measurement of entanglement was performed later, using a two-photon interferometer constructed to verify timeenergy entanglement [20]. Structures such as VOA's can be useful in improving the amplitude balance between different optical pathways on a microchip circuit. VOA's in silicon photonics can be tuned rapidly (at sub nanosecond speeds) are also useful to protect sensitive detectors from unwanted levels of input optical power, such as the so-called "blinding attacks" on single-photon avalanche diodes.

The filters in this device are tuned based on the thermo-optic effect, which is quite strong in silicon photonics. Electronic controllability is also of interest, particularly in the coupled-microring structure since individual resonators can be tuned precisely [21]. Moreover, in contrast to thermal tuning, carrier-driven effects can achieve sub-nanosecond speeds of varying the optical index and absorption. Even faster effects can be achieved using ferro-electric materials which decouple the amplitude and phase effects. For example, opticallytransparent thin-film ferro-electric materials bonded to silicon waveguides can be used to achieve phase shifters with more than $100 \mathrm{GHz}$ of bandwidth, as was demonstrated later [22]. 
Microring resonators are sensitive to disorder. As a way to compensate for defects, we studied potential methods for precisely tuning resonators without heaters. Our method is based on field-induced local oxidation of $\mathrm{Si}_{\text {to }} \mathrm{SiO}_{2}$ via a chemical reaction near an electrically-biased conducting atomic-force microscope tip [23]. A single silicon microresonator can be monitored and controlled more easily [24] than a device that consists of many coupled resonators. Infrared imaging can be used to study light propagation through these structures [15], [25]. Figure 4 shows an example of measuring the drop and add response of a microring filter, using light collected by infrared imaging from a portion of the chip. Although there are some ripples of magnitude about 1-2 $\mathrm{dB}$ in the measurement, essentially the same information about the center frequency, free-spectral range $(5.4 \mathrm{THz})$ and bandwidth is obtained this way as by using edge- or grating-coupled fibers.

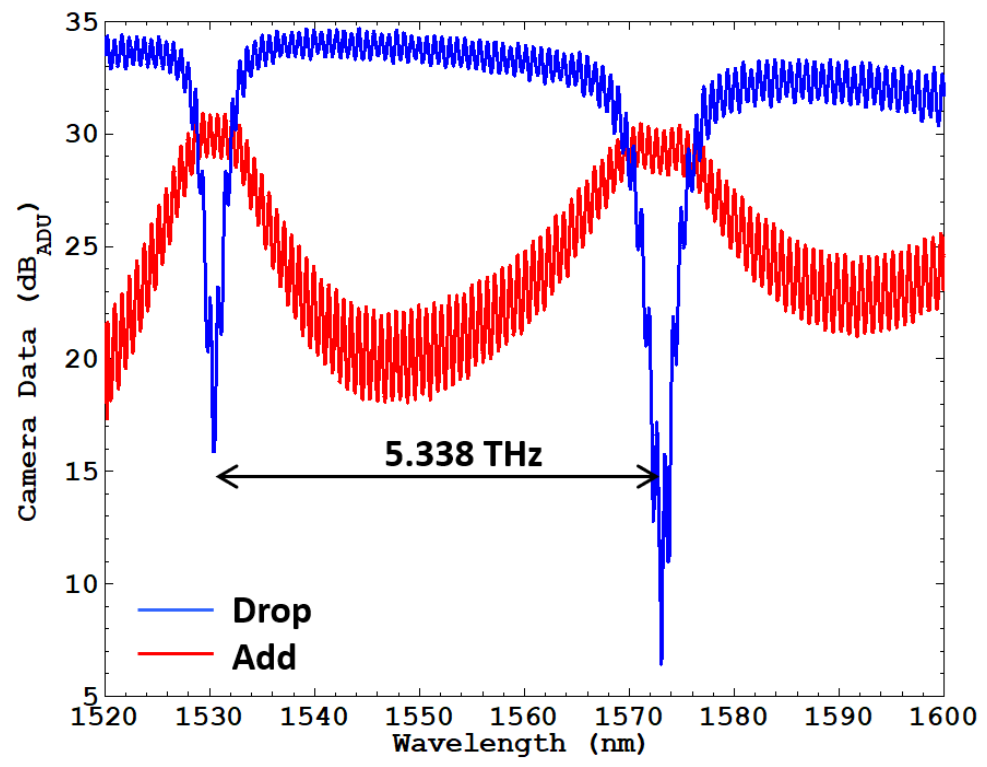

Wafer 15, Chip 6

Figure 4 Measurements of the drop and add functionality of a microring filter using light collected by infrared imaging through a microscope objective.

\section{Open-Access Reporting Initiative}

PRAISE: This open-access document is provided in support of our PRAISE

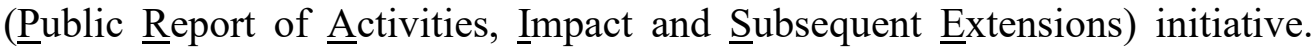
What is it? An open-access document shared with the public which describes the research outcomes of publicly-funded projects. For us, these projects are typically funded by the NSF (National Science Foundation). 


\section{References}

[1] R. Aguinaldo et al., "Silicon photonic integrated components for add, drop, and VOA in a 4-channel data-center network," in 2014 IEEE Photonics Conference, San Diego, CA, Dec. 2014, pp. 1-2. doi: 10.1109/IPCon.2014.7092976.

[2] R. Aguinaldo et al., "Wideband silicon-photonic thermo-optic switch in a wavelength-division multiplexed ring network," Opt. Express, vol. 22, no. 7, p. 8205, Apr. 2014, doi: 10.1364/OE.22.008205.

[3] R. Aguinaldo, Yiran Shen, and S. Mookherjea, "Large Dispersion of Silicon Directional Couplers Obtained via Wideband Microring Parametric Characterization," IEEE Photon. Technol. Lett., vol. 24, no. 14, pp. 1242-1244, Jul. 2012, doi: 10.1109/LPT.2012.2198639.

[4] J. R. Ong, R. Kumar, and S. Mookherjea, "Silicon microring-based wavelength converter with integrated pump and signal suppression," Opt. Lett., vol. 39, no. 15, p. 4439, Aug. 2014, doi: 10.1364/OL.39.004439.

[5] J. R. Ong, R. Kumar, and S. Mookherjea, "Ultra-High-Contrast and Tunable-Bandwidth Filter Using Cascaded High-Order Silicon Microring Filters," IEEE Photon. Technol. Lett., vol. 25, no. 16, pp. 1543-1546, Aug. 2013, doi: 10.1109/LPT.2013.2267539.

[6] S. Mookherjea and A. Yariv, "Pulse propagation in a coupled resonator optical waveguide to all orders of dispersion," Phys. Rev. E, vol. 65, no. 5, p. 056601, Apr. 2002, doi: 10.1103/PhysRevE.65.056601.

[7] S. Mookherjea and A. Yariv, "Kerr-stabilized super-resonant modes in coupled-resonator optical waveguides," Phys. Rev. E, vol. 66, no. 4, p. 046610, Oct. 2002, doi: 10.1103/PhysRevE.66.046610.

[8] S. Mookherjea and A. Yariv, "Optical pulse propagation and holographic storage in a coupled-resonator optical waveguide," Phys. Rev. E, vol. 64, no. 6, p. 066602, Nov. 2001, doi: 10.1103/PhysRevE.64.066602.

[9] S. Mookherjea, D. S. Cohen, and A. Yariv, "Nonlinear dispersion in a coupled-resonator optical waveguide," Opt. Lett., vol. 27, no. 11, p. 933, Jun. 2002, doi: 10.1364/OL.27.000933.

[10] S. Mookherjea, "Using gain to tune the dispersion relation of coupledresonator optical waveguides," IEEE Photon. Technol. Lett., vol. 18, no. 5, pp. 715-717, Mar. 2006, doi: 10.1109/LPT.2006.871144.

[11] M. L. Cooper and S. Mookherjea, "Numerically-assisted coupledmode theory for silicon waveguide couplers and arrayed waveguides," Opt. Express, vol. 17, no. 3, p. 1583, Feb. 2009, doi: 10.1364/OE.17.001583.

[12] S. Mookherjea, "Spectral characteristics of coupled resonators," $J$. Opt. Soc. Am. B, vol. 23, no. 6, p. 1137, Jun. 2006, doi: 10.1364/JOSAB.23.001137. 
[13] J. R. Ong et al., "Low-power continuous-wave four-wave mixing in silicon coupled-resonator optical waveguides," Opt. Lett., vol. 36, no. 15, pp. 2964-2966, 2011.

[14] M. L. Cooper et al., "235-ring Coupled-Resonator Optical Waveguides," in Conference on Lasers and Electro-Optics 2010, San Jose, California, 2010, p. CTuHH3. doi: 10.1364/CLEO.2010.CTuHH3.

[15] M. L. Cooper, G. Gupta, J. S. Park, M. A. Schneider, I. B. Divliansky, and S. Mookherjea, "Quantitative infrared imaging of silicon-on-insulator microring resonators," Opt. Lett., vol. 35, no. 5, p. 784, Mar. 2010, doi: 10.1364/OL.35.000784.

[16] S. Mookherjea and M. A. Schneider, "Avoiding bandwidth collapse in long chains of coupled optical microresonators," Opt. Lett., vol. 36, no. 23, p. 4557, Dec. 2011, doi: 10.1364/OL.36.004557.

[17] M. L. Cooper and S. Mookherjea, "Modeling of Multiband Transmission in Long Silicon Coupled-Resonator Optical Waveguides," IEEE Photon. Technol. Lett., vol. 23, no. 13, pp. 872-874, Jul. 2011, doi: 10.1109/LPT.2011.2141657.

[18] J. R. Ong and S. Mookherjea, "Quantum light generation on a silicon chip using waveguides and resonators," Opt. Express, vol. 21, no. 4, p. 5171, Feb. 2013, doi: 10.1364/OE.21.005171.

[19] S. Mookherjea, "Semiconductor coupled-resonator optical waveguide laser," Appl. Phys. Lett., vol. 84, no. 17, pp. 3265-3267, Apr. 2004, doi: 10.1063/1.1719278.

[20] R. Kumar, M. Savanier, J. R. Ong, and S. Mookherjea, "Entanglement measurement of a coupled silicon microring photon pair source," Opt. Express, vol. 23, no. 15, p. 19318, Jul. 2015, doi: 10.1364/OE.23.019318.

[21] S. Mookherjea, J. R. Ong, X. Luo, and L. Guo-Qiang, "Electronic control of optical Anderson localization modes," Nature Nanotech, vol. 9, no. 5, pp. 365-371, May 2014, doi: 10.1038/nnano.2014.53.

[22] X. Wang, P. O. Weigel, J. Zhao, M. Ruesing, and S. Mookherjea, "Achieving beyond-100-GHz large-signal modulation bandwidth in hybrid silicon photonics Mach Zehnder modulators using thin film lithium niobate," APL Photonics, vol. 4, no. 9, p. 096101, Sep. 2019, doi: $10.1063 / 1.5115243$.

[23] Y. Shen, I. B. Divliansky, D. N. Basov, and S. Mookherjea, "Perfect set-and-forget alignment of silicon photonic resonators and interferometers," in Optical Fiber Communication Conference/National Fiber Optic Engineers Conference 2011, Los Angeles, California, 2011, p. PDPC3. doi: 10.1364/OFC.2011.PDPC3.

[24] M. Savanier, R. Kumar, and S. Mookherjea, "Optimizing photon-pair generation electronically using a $p-i-n$ diode incorporated in a silicon microring resonator," Appl. Phys. Lett., vol. 107, no. 13, p. 131101, Sep. 2015, doi: 10.1063/1.4932047. 
[25] S. Mookherjea and H. R. Grant, "High dynamic range microscope infrared imaging of silicon nanophotonic devices," Opt. Lett., vol. 37, no. 22, p. 4705, Nov. 2012, doi: 10.1364/OL.37.004705. 